\title{
Measurement of Parameters of the Optic Disk in Ophthalmoscopic Color Images of Human Retina
}

\author{
Edgardo M. Felipe Riverón ${ }^{1}$ and Mijail del Toro Céspedes ${ }^{2}$ \\ ${ }^{1}$ Center for Computing Research, National Polytechnic Institute, \\ Juan de Dios Batiz s/n, P.O. 07738, Mexico \\ edgardo@cic.ipn.mx \\ ${ }^{2}$ Havana University, Havana, Cuba \\ mijail_dt@yahoo.es, mijail@ausa.gae.com.cu
}

\begin{abstract}
The objective of this paper is to measure some important parameters of the optic disk (or optic papilla) in ophthalmoscopic color images of human retinas. The approach consists of locating the optic disk automatically, segmenting its contour and the contour of the depression-like feature caused by glaucoma, called an excavation or cup. Then the corresponding areas are measured to calculate the ratio Cup/Disc and the relative displacement of the centroids of both regions. To achieve these objectives, noise is filtered, luminance is normalized, and a thresholding technique is used. The results obtained will aid the work of ophthalmologists by increasing the quality of automatic diagnosis of glaucoma, one of the main causes of blindness worldwide.
\end{abstract}

\section{Introduction}

There are three visible structural anatomical elements present in the rear pole of the retina: the macula, the optic disk (or optic papilla), and the vascular network, composed of the thicker and darker red vein network, and the thinner arterial network of clearer reddish tone (Fig. 1).

The optic disk or papilla is the clearest area in images of the rear pole of the retina. In a normal papilla, the vascular network coming out from the choroids travels through the center of the nervous fibers that constitute the optic nerve, which travels through a tube-like structure toward the brain. Due to glaucoma, caused frequently by an abnormal increase of the intraocular pressure, an excavation (or cup) is created in the papilla. This excavation produces a thickening of the wall of the papilla, which moves the cluster of veins and arteries toward the nasal side of the affected eye (Fig. 2). In time, the optic nerve is damaged, causing first loss of peripheral vision and later complete loss of vision in those patients suffering from this disease.

The papilla and the excavation areas in the rear pole constitute the objects and areas of interest (AOI) in this work. Figure 2 shows how the thickening of the wall of the papilla assumes a yellowish coloration, while the excavation stands out even more. The thick area of orange coloration is called the neural ring.

Glaucoma is one of the most prevalent illnesses of the retina in the world and one that, if not diagnosed on time, often leads to total blindness [1]. More than 66 million people in the world are affected by glaucoma [2]. The search for computerized image processing techniques to help diagnose this illness is an area of considerable interest. The work reported in this paper is a step in that direction. 


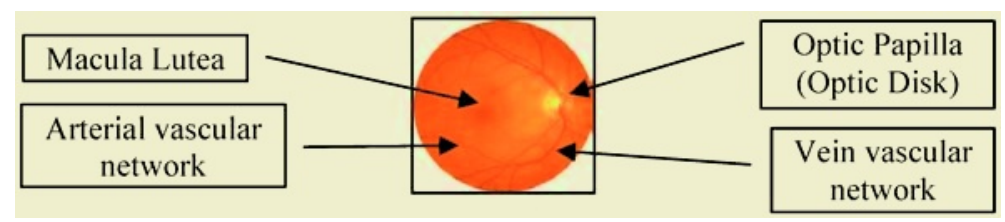

Fig. 1. Anatomic structural elements of the retina

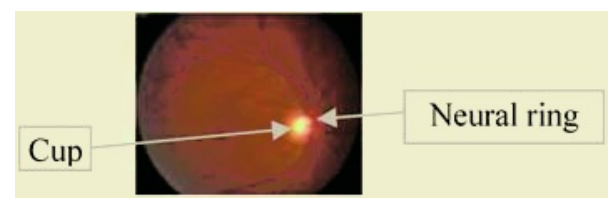

Fig. 2. Image of the optic disk or papilla in a human retina with glaucoma

\section{The Problem}

Our task deals with the localization and isolation of the optic disk in images of the retina. Any strategy applied to achieve this purpose should be independent of lack of uniformity in illumination, and be invariant to translation, rotation and scale in the images being analyzed. In the method described in the following sections, the border of the optic disk and that of the excavation mentioned earlier are segmented, and the position of their centroids is calculated. Finally, a relative shift between centroids and the ratio Cup/Disk of corresponding areas are computed, from which ophthalmologists are given an objective index of the presence or absence of glaucoma.

\section{Previous Works}

The successful localization and segmentation of the optic disk in images of the rear pole of human retina has been treated previously in the literature. Different methods like tracking of the optic disk have been described through a pyramidal decomposition and search of the contour based on the Hausendorff distance, where the region of the optic disk is found by means of multiscale analysis or of a pyramidal approach using a simple wavelet transformation [3]. The automatic localization of the papilla has also been achieved by means of the analysis of principal components. This technique is based on groupings by regions the brightest of pixels in the image [4].

Because the papilla is nearly elliptical, the Hough Transform has been applied also to the detection of this type of shape [5].

The border of the optic disk has also been detected by means of active contours (snakes), which simulate the adjustment from an elastic curve to the borders of the objects of interest in an image [7]. This has been applied in the detection of the optic disk in retina images, where they combine a priori knowledge about the papilla and its approximate size, keeping in mind the individual characteristics of the image.

\section{Sequence of Operations}

All color images used in this work and reported here were obtained from the same source and have $300 \mathrm{dpi}$ of spatial resolution, $24 \mathrm{bits} / \mathrm{pixel}$ and were normalized to a size of 720 x 576 pixels. In total there were 107 images from 34 cases. 
The operations carried out during the complete process were:

- Automatic localization of the papilla.

- Determination of centroids and reduction in size the image.

- Preprocessing (homogenizing gray levels of the background and noise cleaning).

- Measurements.

\subsection{Automatic Localization of the Papilla}

Because the color images used in this work are represented in the RGB color model, the papilla is located automatically by means of the thresholding method of Otsu [8] [9] from the green plane of the image. With a binary opening using a flat disk structure element (SE) of 5 pixels of diameter, the isolated components were cleaned and the small basins inside the AOI were filled using a binary closing operation.

\subsection{Determination of the Centroids and Reduction of the Image}

All images in this work are considered isotropic, that is, with objects having uniform characteristics in all dimensions. This allows us to compute easily the position of centroids. To obtain the coordinates of a centroid, we applied an external border morphological operation with a flat cross shape SE of $3 \times 3$ pixels. We then computed the centroid of the resulting convex polygon using the following general expressions for the area (Eq. 1) and for the coordinates X and Y (Eqs. 2 and 3) shown in Table 1 [10].

$\mathrm{Xi}$ and $\mathrm{Yi}$ are the components $\mathrm{x}$ and $\mathrm{y}$ of pixel $\mathrm{i}$ in the polygon of $\mathrm{N}$ pixels. The only restriction to using the formula for computing the area in the case of several polygons is that there can be no overlap among them, a condition that is generally satisfied in practice.

Table 1. Expressions for calculating the position of the centroid

$$
\begin{gathered}
A=\frac{1}{2} \sum_{i=0}^{N-1}\left(X_{i} Y_{i+1}-X_{i+i} Y_{i}\right) . \text { Polygon area. } \\
C_{x}=\frac{1}{6 A} \sum_{i=0}^{N-1}\left(X_{i}+X_{i+1}\right)\left(X_{i} Y_{i+1}-X_{i+1} Y_{i}\right) \cdot \text { X Component. } \\
C_{y}=\frac{1}{6 A} \sum_{i=0}^{N-1}\left(Y_{i}+Y_{i+1}\right)\left(X_{i} Y_{i+1}-X_{i+1} Y_{i}\right) . \text { Y Component. }
\end{gathered}
$$

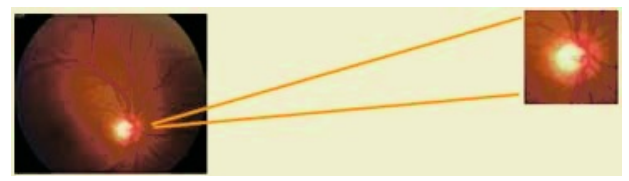

Fig. 3. Automatic localization of the papilla 
After the position of the centroid is calculated, we proceed to crop the original color image from the original $720 \times 576$ pixels to $200 \times 200$ pixels around the centroid, which guarantees that the optic disk is contained completely in the subimage. This subimage is our AOI (Fig. 3).

\subsection{Homogenizing Gray Levels of the Background}

In general, the capture of images was not focused directly toward the papilla. For that reason the area where it is located in an image is not of uniform illumination. To compensate for this, an algorithm to homogenize the background was applied to facilitate the subsequent segmentation by thresholding (Fig. 4).

The located subimage was subdivided into $\mathrm{k} x \mathrm{k}$ cells, and on the basis of the average of gray levels of the corresponding pixels (Eqs. 4a-4d), it was determined which of them was the darkest (Eq. 5). After this, a calculation is performed (Eq. 6) to determine how many levels missed each pixel of remaining grids to be equally dark than the average of the darkest. Equation 7 shows the expression used to calculate the magnitude to be added to each pixel of each grid to achieve the homogenizing (See Table 2).

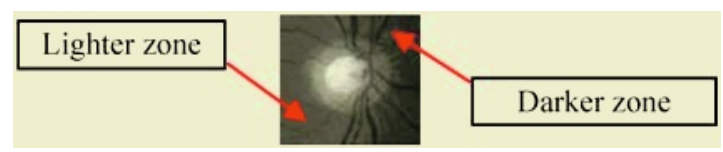

Fig. 4. Darker and lighter zones around the optic papilla

Table 2. Expressions for homogenizing the uneven background

$$
\begin{aligned}
& S_{(0,0)}=\sum_{i=0}^{w c} \sum_{j=0}^{h c} g_{i j} \\
& S_{(0, k-1)}=\sum_{i=0}^{w c} \sum_{j=h-h c}^{h} g_{i j} \\
& s_{(k-1,0)}=\sum_{i=w-w c}^{w} \sum_{j=0}^{h c} g_{i j} \\
& s_{(k-1, k-1)}=\sum_{i=w-w c}^{w} \sum_{j=h-h c}^{h} g_{i j} \\
& S=\min \left(S_{(0,0)}, S_{(0, k-1)}, S_{(k-1,0)}, S_{(k-1, k-1)}\right) \\
& v_{m, n}=\frac{S-S_{(i, j)}}{w C \cdot h C} \quad i, j=\{0, k-1\} \\
& v_{(m, n)}=\frac{\left(v_{(m, k-1)}-v_{(m, 0)}\right) n}{k-1}+v_{(m, 0)}: 0<n<k-1,0 \leq m \leq k-1
\end{aligned}
$$


In our case the size of the subdivision selected was $20 \times 20$ cells (Fig. 5). To eliminate the noise in images, a Gaussian filter was applied with a flat square window of $5 \times 5$ pixels and standard deviation $\sigma=0.8$ pixels, followed by an averaging filter with a flat square window of the same size [5] [12].
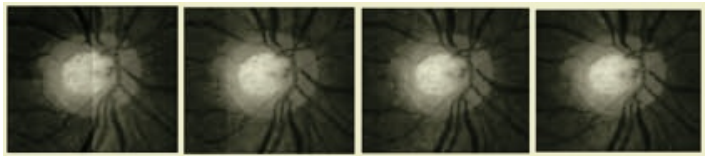

Fig. 5. Results of the homogenizing for $2 \times 2,4 \times 4,10 \times 10$, and $20 \times 20$ cells

\subsection{Segmentation of Contours of the Papilla and the Excavation}

Finally, the best results to segment the papilla and the excavation were achieved by a strategy that combines segmentation by thresholding according to Otsu [8], and the sequential application of binary opening, closing and external border. This procedure we called OACE, formed by the Spanish initials of Otsu, Opening, Closing and External border (Fig. 6). When using Otsu's method to threshold an image, a value $\varepsilon$ taken from the histogram is needed to regulate the level of the total variation of the sample. In this study the value $\varepsilon$ oscillated in the range [0.1-0.2] with a mean value of 0.15 for the papilla, and between [0.03-0.06] with a mean value 0.045 for the segmentation of the excavation area. They proved to be adequate in most of cases.

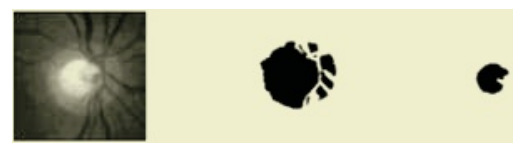

Fig. 6. Thresholding by Otsu with $\varepsilon=0.15$ for the papilla and $\varepsilon=0.05$ for the excavation

When detecting the contour of the papilla in binary images, it was observed that in some cases there were present isolated small spurious components (shown by arrows in Fig. 7a). To eliminate these components an opening was applied with a flat disk of 5 pixels in diameter. To restore the original image (now without the artifacts) a reconstruction operation by binary dilation with a similar SE was used.

(a)

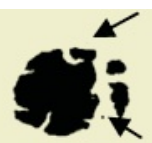

(b)

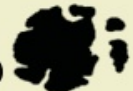

(c)

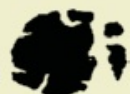

Fig. 7. (a) Thresholded image. (b) Binary opening. (c) Reconstruction by binary dilation

The vascular cluster present inside the papilla causes in the binary images after segmentation the separation among the components of the papilla and the small gulfs in the excavation. To enhance the shape of these components a closing was applied with flat disks SE of different sizes: 13 pixels for the papilla and 43 pixels for the excavation (Figs. 8c and 8f). 
(a)

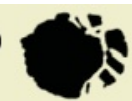

(b)

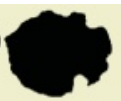

(c)

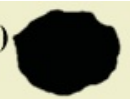

(d)

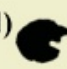

(e)

(f)

Fig. 8. (a) and (d) Papilla and excavation after Otsu. (b) and (e) After closing with a flat disk of 13 pixels of diameter. (c) and (f) Idem with a flat disk of 43 pixels of diameter

Subsequently, to extract the external borders of the papilla and the excavation, the morphological operation External Border was applied with a flat cross SE of $3 \times 3$ pixels, as shown in Fig. 9. Figure 10 shows both contours overlapping separately the original image.

(a)

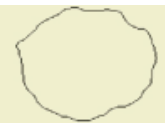

(b)

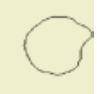

Fig. 9. (a) External border of the papilla. (b) Idem of the excavation

(a)

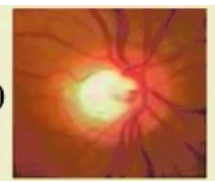

(b)

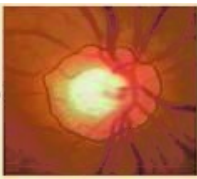

(c)

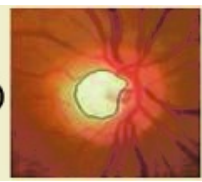

Fig. 10. Contours after applying OACE. (a) Original image. (b) $\varepsilon=0.17$. (c) $\varepsilon=0.052$

\subsection{Measurements}

By means of the Equation 1, the area occupied by the papilla and excavation was calculated in pixels. To determine the coordinates of the centroids, Equations 2 and 3 were used, starting from the corresponding contours. Because of the papilla has a nearly elliptic shape, the contour was approximated by an ellipse (Fig. 11).

One form to detect the presence of the glaucoma is through the so called Cup / Disk ratio, where the excavation is not more than the sinking that takes place in the papilla with respect to the normal level of the retina. The relationship Cup / Disk offers us a normal / abnormal index, based on the division of the optic disk in 10 sections, starting from the geometric center of the ellipse that bounds the optic disk.

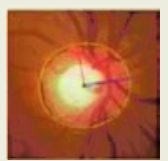

Fig. 11. Ellipse that is better adjusted to the papilla

From the ellipses that better adjust the papilla (EExt) they were calculated (Fig. 12a):

- The Euclidean distances in pixels from the border of the excavation (blue), until the border of the ellipse EExt (red), in N, S, E and W directions [13].

- The Cup /Disk ratio.

- The distances between centroids of the two areas occupied by the papilla and the excavation, to obtain the displacement that takes place among them.

Global results are shown in Table 3 for 107 glaucomatous images analyzed. The percentage of effectiveness was of $94 \%$. 
Table 3. Results obtained when OACE method was applied to 107 glaucomatous images

\begin{tabular}{|c|c|}
\hline Task & Images \\
\hline Optic disk detection & 101 \\
\hline Excavation detection & 105 \\
\hline Both contours & 97 \\
\hline
\end{tabular}

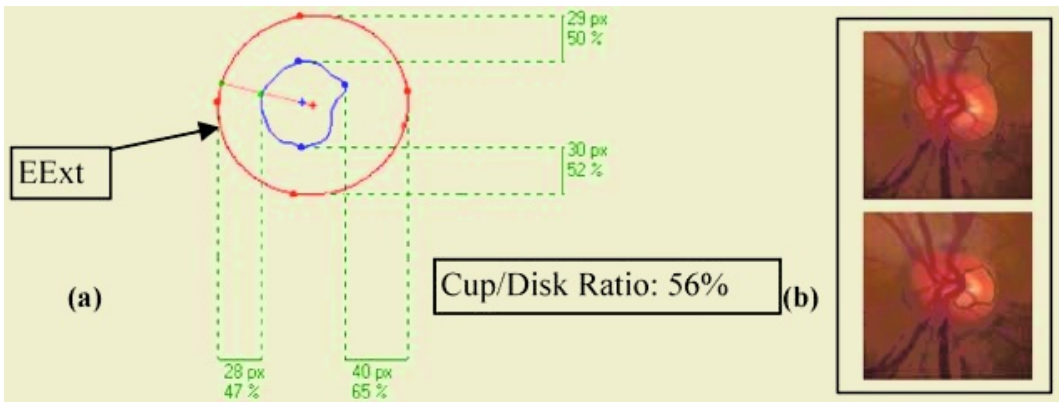

Fig. 12. (a) Indication and results of the measurements. (b) Two cases where OACE failed

\section{Conclusions}

This work has been oriented toward the measurements of parameters of the optic disk in real images of human retinas. The optic disk was located automatically, and its contour and the contour of any excavations present were segmented in order to measure the areas and relative displacements between correspondent centroids, as the main parameters indicative of the presence of glaucoma. A homogenizing algorithm was applied to equalize the illumination of the areas surrounding the papilla. Gaussian and averaging filters were used to reduce the random noise present in the original images. For the detection of contours of the papilla and the excavation, we used a strategy that combines segmentation using the thresholding method of Otsu, together with the binary operators of opening, closing and external border. The areas of the papilla and the excavation were measured, as were the relative displacement of correspondent centroid. Finally, the contour of the papilla was approximated by an ellipse to calculate the desired relationship Cup / Disk.

\section{References}

1. Alemañy Martorell, J., Marrero Faz, E., Villar Valdés, R., Oftalmología. Capítulo 14 (1980).

2. Lubinus Badillo, F. G., Mantilla Suárez, J. C., Valencia, A., Rueda, J. C., Estudio de la circulación retrobulbar con imagen doppler a color en pacientes con glaucoma asimétrico (2002).

3. Gagnon, L., Lalonde, M., Beaulieu, M., Boucher, M.-C. Procedure to detect anatomical structures in optic fundus images, Computer Research Institute of Montreal; Dept. of Ophthalmology, Maisonneuve-Rosemont Hospital (2001).

4. Li H. Chutatape O., Automatic Location of Optic Disk in Retinal Image. School of Electrical and Electronic Engineering (1999). 
5. Molina, R., Introducción al Procesamiento y Análisis de Imágenes Digitales, en http://www-etsi2.ugr.es/depar/ccia/mia/complementario/

Procesamiento_Imagenes/node14.html

6. McLaughlin, R. A., Technical Report - Randomized Hough Transform: Improved Ellipse Detection with Comparison (1995).

7. Mendels, F., Heneghan, C., and Thiran, J. P., Identification of the Optic disk boundary in retinal images using active contours (2000).

8. Otsu, N., A threshold Selection Method from Gray-Level Histogram. IEEE Trans Systems, Man and Cybernetics SMC-9:62-66 (1976).

9. Rodriguez, R. and Alarcon, T. E, Color Segmentation Applied to the Study of the Angiogenesis (2002).

10. Vincent, L., Componentes de SIGAU, Capítulo 5 (1999).

11. Vincent, L., Morphological grayscale Reconstruction in Images Analysis: Applications and Efficient Algorithms, IEEE Transactions on Images Processing, Vol 2, pp. 176-201 (1993).

12. Image Processing Learning Resources: www.dai.ed.ac.uk\HIPR $2 \backslash$ mean.htm

13. Felipe-Riverón, E. M., Medidas de distancias, curso sobre Conceptos básicos sobre procesamiento de imágenes, CIC-IPN (2002). 